Oper Orthop Traumatol 2014 · 26:112-113

DOI 10.1007/s00064-014-0298-z

Online publiziert: 2. April 2014

๑) Springer-Verlag Berlin Heidelberg 2014

D.C. Wirtz

Klinik und Poliklinik für Orthopädie und Unfallchirurgie, Universitätsklinikum Bonn

\title{
Periprothetische Knochendefekte am Hüftgelenk
}

stützschalen dargestellt, wobei als wesentliche Voraussetzungen zur Anwendung der Methode ein stabiler lateraler Pfannenerker und dorsaler Azetabulumpfeiler anzusehen sind, so dass die Abstützschale „kippelfrei“ fixiert werden kann. Ist dies bei ausgedehnteren Azetabulumdefekten nicht mehr gegeben, kann die von Günther et al. beschriebene Technik des „Augment-andCage“-Systems angewendet werden. Dabei wird der laterale Pfannenerkerdefekt durch ein Metallaugment ersetzt und der mit Knochentransplantaten aufgefüllte zentrale Defekt mit einer überbrückenden ilioischialen Abstützschale geschützt. Als Alternative zu diesen beiden Techniken wird von Petheram und Howell die weithin als "Exeter-Methode“ bekannte Vorgehensweise des „impaction grafting" und Einzementieren einer Polyethylenpfanne dargestellt. Dabei ist sowohl am Azetabulum als auch am Femur - wie im Beitrag von Heyligers dargestellt - die operative Technik der impaktierenden Knochenrekonstruktion entscheidend für die Ergebnisqualität mit Umbau der Transplantate und langfristig stabiler Prothesenfunktionalität. Gerade am Femur gibt es jedoch auch solch ausgedehnte Defektsituationen, dass ein „Impaction Grafting“ aufgrund „fehlendem kortikalem Rahmen“ praktisch nicht mehr möglich ist. In solchen Situationen bietet, wie von Barden et al. dargestellt, die Anwendung von sog. „Strut-Grafts“ am Femur eine Möglichkeit, möglichst biologisch zu rekonstru- ieren und die ansonsten nur noch bestehende Tumorprothesenimplantation $\mathrm{zu}$ vermeiden.

Zusammenfassend bleibt festzuhalten, dass es in der Rekonstruktion von periprothetischen Knochendefekten am Azetabulum und Femur nicht eine Methode gibt, die für jede Situation und alle Patienten angewendet werden sollte. Vielmehr sind unter den oben genannten Kriterien die verschiedenen operativen Techniken komplementär im Sinne eines patienten- und defektadaptierten Algorithmus anzuwenden. Als verantwortlicher Herausgeber dieses Schwerpunkthefts wünsche ich Ihnen viel Spaß beim Lesen und einen erträglichen Erkenntnisgewinn für Ihre tägliche klinische Arbeit.

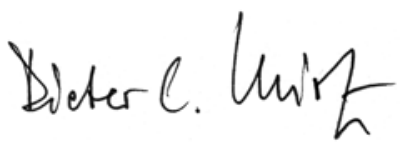

Dieter C. Wirtz

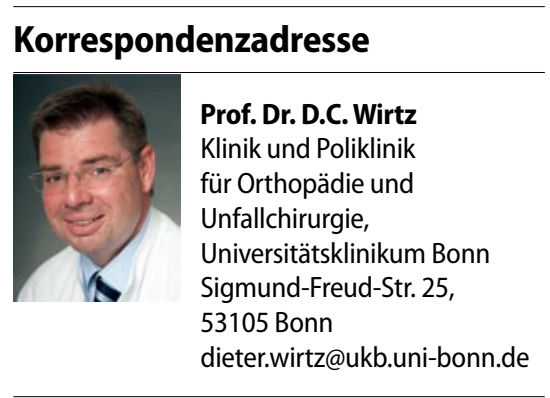

\section{In der Arbeit von Friedrich et al. wird die Technik des „Impaction Grafting“ bei Verwendung von überbrückenden $\mathrm{Ab}$ - \\ 1) Bei periprothetischen Knochendefekten am Hüftgelenk eignet sich nicht jede Rekonstruk- tionsmethode für alle Situationen}

\footnotetext{
Operative Orthopädie und Traumatologie $2 \cdot 2014$
} 


\section{Einhaltung ethischer Richtlinien}

Interessenkonflikt. D.C. Wirtz gibt an, dass kein Interessenkonflikt besteht.

\section{Literatur}

1. Eingartner C, Volkmann R, Ochs U et al (2006) Intramedulläre Stabilisierung periprothetischer Femurfrakturen unter besonderer Berücksichtigung des vorbestehenden Knochendefekts. Oper Orthop Traumatol 18:341-363

2. Mumme T, Müller-Rath R, Andereya S, Wirtz DC (2007) Zementfreier Femurschaftwechsel mit de Modularen Revisions Prothese MRP-Titan-Revisionsschaft. Oper Orthop Traumatol 19:56-77

3. Fink B, Grossmann A (2008) Technik der Implantation einer zementlosen Pressfit-Pfanne bei Pfannenrevisionen mit größeren Knochendefekten. Oper Orthop Traumatol 20:157-167

4. Halder A, Beier A, Neumann W (2009) Rekonstruktion des Azetabulums mit strukturiertem Knochentransplantat in Press-fit-Technik. Oper Orthop Traumatol 21:232-240

5. Rudert M, Holzapfel BM, Kratzer F, Gradinger $R$ (2010) Das Kranialpfannensystem zur defekttypassoziierten Therapie von Azetabulumdefekten. Oper Orthop Traumatol 22:241-255

6. Fink B, Grossmann A, Sebena P (2010) Pfannenwechsel bei größeren Defekten mit Antiprotrusionsringen. Oper Orthop Traumatol 22:256-267

\section{Ausschreibung Oskar-Medizinpreis 2014}

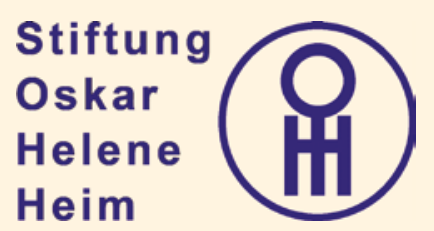

Die Stiftung Oskar-Helene-Heim fördert die Wissenschaft und Forschung auf dem Gebiet der Medizin. Als besonderes Förderprojekt verleiht die Stiftung jährlich den mit 50.000 Euro dotierten Oskar-Medizinpreis. Mit diesem Medizinpreis werden hervorragende Leistungen gewürdigt und die Weiterführung von Forschungen unterstützt.

Im Jahr 2014 wird dieser Medizinpreis auf dem Gebiet der

\section{Orthopädie und Unfallchirurgie}

ausgeschrieben. Der Gelenkersatz gehört zu den häufigsten Operationen in deutschen Krankenhäusern. Daher ist die Qualitätsverbesserung bei der Endoprothetik eine ständige Herausforderung. Es geht um die Optimierung des operativen Zugangs und die sichere Platzierung der Implantate, die Reduktion von Komplikationen und die schnelle postoperative Rehabilitation der Patienten. Die erhebliche volkswirtschaftliche Relevanz gebietet es, die Qualität und Patientensicherheit in der Endoprothetik zu verbessern. Letztlich ist ein wichtiger Maßstab die Erfassung des individuellen Nutzens, den die Patienten von der Behandlung wahrnehmen.

Die Hüftendoprothetik war in den vergangenen Jahren der häufigste Eingriff und wird als die erfolgreichste orthopädische Operation eingeschätzt. Die Knieendoprothetik hat inzwischen mengenmäßig zu den Hüftoperationen aufgeschlossen und wird von der fachlichen Anforderung an das klinische Vorgehen deutlich anspruchsvoller beurteilt. Mit dem Oskar-Medizinpreis 2014 soll daher ein/e habilitierte/r Mediziner/in ausgezeichnet werden, die/der einen relevanten Beitrag zum Thema

„Verbesserung von Qualität und Patientensicherheit beim elektiven Hüft- und/oder Kniegelenkersatz" in der Grundlagen- und/oder klinischen Forschung in Deutschland leistet. Fachübergreifende Forschungsgruppen sind ebenfalls zur Bewerbung zugelassen.

Der prämierte Erkenntnisgewinn soll einer breiten Öffentlichkeit vermittelbar sein.

Dem Antrag sollen der Lebenslauf, die fünf wichtigsten Arbeiten aus den letzten drei Jahren zum Thema „Innovation und Patientensicherheit bei der elektiven Hüft- und/oder Knieendoprothetik (Primärversorgung und Wechselendoprothetik)" sowie eine inhaltliche Zusammenfassung der Forschungsergebnisse beigefügt werden, ergänzt um weitere Forschungsplanungen. Arbeiten, die bereits eine anderweitige Prämierung erhalten haben, sind nicht zugelassen.

Das Preisgeld ist für Forschungszwecke nach der freien Entscheidung des Preisträgers zu verwenden. Der Preisträger hat der Stiftung die Verwendung in geeigneter Weise zu belegen.

Der Antrag ist bis zum 31. Mai 2014 bei der Stiftung Oskar-Helene-Heim einzureichen (info@stiftung-ohh.de). Weitere Informationen erteilt der Geschäftsführer der Stiftung, Werner Ukas: werner.ukas@stiftung-ohh.de, Tel. 030 8102-1100.

Zweck der Stiftung Oskar-Helene-Heim ist die Förderung von Wissenschaft und Forschung in der Medizin, insbesondere der Orthopädie, der Lungenheilkunde, Gastroenterologie und Viszeralchirurgie sowie der Orthopädietechnik. Zu diesem Zweck unterstützt die Stiftung Forschungsprojekte oder sonstige gemeinnützige gesundheitsfördernde Vorhaben, verleiht Stipendien und vergibt jährlich den Oskar-Medizinpreis und die Helene-Medaille.

Quelle: Stiftung Oskar-Helene-Heim, www.stiftung-ohh.de 